\title{
Short Letrozole Therapy Versus Extended (Long) Letrozole Therapy For Induction of Ovulation in Women With Polycystic Ovary Syndrome. Randomised study
}

\author{
M.A.Mohammed, K.M.Salama, B.E.Sakr and S.A.Azab \\ Obstetrics and gynecology, Dept.,Faculty of Medicine, Benha Univ., Benha, Egypt \\ E-Mail:saad12@gmail.com
}

\begin{abstract}
Presentation: Polycystic Ovary Syndrome is quite possibly the most well-known reasons for anovulatory fruitlessness, influencing $8-13 \%$ of conceptive matured ladies . It is by a long shot the most well-known reason for hyperandrogenic anovulatory fruitlessness and was depicted the greater part a century prior, the basic reason for this issue is as yet dubious. Point: to examine the viability of short(2.5mg day by day for 5 days) versus broadened course (2.5mg day by day for 10 days) of letrozole treatment for ovulation acceptance in ladies with polycystic ovary syndrome.Subject \&Method: This examination directed on (40) patients with PCOS They were arbitrarily assigned in two gatherings. Gathering 1 (20 patients )got letrozole in adose of $5 \mathrm{mg}$ for 5 days while the gathering 2 (20 patients) got letrozole in adose of $2.5 \mathrm{mg}$ for 10 days.Result : The all out number of follicles in the present moment letrozole was $3 \pm 1$ while it was $4 \pm 1$ in the long letrozole bunch. In our examination the endometrial thickness was $8 \pm 2$ in both short and long letrozole bunch . The pregnancy rate was $15 \%$ in short letrozole bunch while it was $25 \%$ in long letrozole bunch . End: The long letrozole treatment (2.5mg for 10 days) can create more develop follicles and in this manner a greater number of pregnancies than the short letrozole treatment ( $5 \mathrm{mg}$ for 5 days).
\end{abstract}

Keywords: PCOS, Letrozole.

\section{Introduction}

Polycystic ovary condition (PCOS) is a heterogeneous disorder and is thinking about the most well-known endocrine problem in ladies at regenerative age [1].

It was first portrayed in 1935 by Stein and Levanthal (1935) in the American Journal of Obstetrics and Gynecology. However, it stays a condition that is confounding to numerous patients and experts regarding its introduction, work-up, and the executives. There is a range of introducing objections and actual discoveries just as cover with different problems, like the metabolic disorder.

IT is the most widely recognized endocrine problem of conceptive matured ladies and influences roughly $4 \%$ to $12 \%$ overall populace studies(9) PCOS is related with $75 \%$ of all anovulatory messes causing fruitlessness, Diagnostic models of PCO PCOS Defined by ESHRE/ASRM (Rotterdam) 2003 as a disorder incorporates two out of the accompanying:

a) Clinical and/or biochemical indications of hyperandrogenism

b) Polycystic ovaries by ultra sound (presence of at least 12 follicles in every ovary estimating 2 to 9 $\mathrm{mm}$ in measurement or potentially expanded ovarian volume $(10 \mathrm{~cm} 3$; determined utilizing the equation $0.5 \mathrm{x}$ length $\mathrm{x}$ width $\mathrm{x}$ thickness). The transvaginal approach ought to be used [12].

With prohibition of different conditions with comparable signs, for example, androgen-discharging tumors or Cushing's disorder and thyroid brokenness and hyperprolactinemia.

CC - obstruction alludes to the failure [10] to ovulate with $150 \mathrm{mg}$ of cc for in any event 3 cycles, while CC-disappointment is characterized as inability to consider with $\mathrm{CC}$ regardless of fruitful ordinary ovulation for 6-9 cycles.
Elective strategies utilized for the ovulation enlistment in PCOS are CC in mix with metformin, $\mathrm{CC}$ in blend with dexamethasone, specific aromatase inhibitor, for example, letrozole and anastrozole, gonadotropins, laproscopic ovarian penetrating.

Aromatase (cytochrome P-450 [CYP]) catalyzes the rate-restricting advance (transformation of steroidal C-19 androgens to C-18 estrogens) in estrogen biosynthesis [14] Aromatization is the last advance in steroid biosynthesis; and, thusly, aromatase is an alluring objective for specific hindrance [7].

Aromatase inhibitors: The quest for intense and particular inhibitors of aromatase began with the original inhibitor aminoglutethimide. Be that as it may, aminoglutethimide needed selectivity for aromatase and hindered biosynthesis of cortisol, aldosterone, and thyroid chemical just as aromatase; additionally, aminoglutethimide was likewise found to incite hepatic proteins .

Second-age AIs incorporated the nonsteroidal inhibitor fadrozole and the steroidal inhibitor formestane (4-hydroxyandrostenedione). Fadrozole was better than aminoglutethimide regarding power, selectivity, and security, yet its selectivity was not finished and clinical preliminaries proposed that it was not any more successful than tamoxifen. To enhance fadrozole, Novartis incorporated a progression of new mixtures. Design action relationship examines were then performed to recognize the most intense AIs from a progression of benzyl-azole subsidiaries of fadrozole [13].

The third-age AIs Letrozole (Femara1) was the consequence of this construction movement way to deal with drug plan and accomplished the exploration objective of making an exceptionally strong and absolutely particular AIs. 


\section{Aim of the work}

The point of this study, to think about the viability of short(5mg every day for 5 days) versus expanded course $(2.5 \mathrm{mg}$ day by day for 10 days) of letrozole treatment for ovulation acceptance in ladies with polycystic ovary disorder.

\section{Subject and methods}

- Type of study: Prospective randomized comparative study .

- Site of study: Obstetrics and Gynecology department, Benha university .

- The study was approved by the Ethics Board of Benha university.

- Sample size: 40 infertile patients diagnosed as having PCO based on Rotterdam criteria (2003),

Groups of the study: patients were randomly allocated in two groups :

- Group 1: short letrozole therapy (20 patients).

- Group 2 : long letrozole therapy (20 patients).

\subsection{Inclusion criteria}

- Age: 18-35 years old.

- Infertility $\geq 2$ years.

- Diagnosis of PCOS according to Rotterdam Criteria.

- Nonsmoker or addict to any substance.

- married.

\subsection{Exclusion criteria}

- History of pelvic surgery or trauma.

- Women with infertility factors other than anovulation.

- Male factor of infertility.

- Smokers, alcohol addict.

- Other causes of hyperandrogenism.

\subsection{All participating patients had the following:}

\section{I-Full history}

- Personal history

- History of present illness

- Menstrual history

- Sexual history

- Past history

- Family history

\section{II-Complete examination}

- General examination

- Abdominal examination
- Local (Pelvic) examination

III-Investigations

- General (CBC, urinalysis, Random blood sugar) when needed.

- Specific: FSH, LH, Prolactin. Androgen,Estradiol,Estrone on day three of menstrual cycle.

\subsection{Methodology}

Patients of short letrozole treatment bunch ( 20 patients) got $5 \mathrm{mg}$ of letrozole (2 tablets of letrozole 2.5mg, Sandoz Pharmaceutical Company, Australia) day by day beginning from day 1 of unconstrained (or progesterone incited, utilizing Medroxyprogesterone acetic acid derivation $5 \mathrm{mg}$ tab, one tab every day for 5 days)

Feminine seeping for 5 days (patients, up to 3 cycle).

Patients of long letrozole treatment group $(20$ patients) got $2.5 \mathrm{mg}$ of letrozole (one tab of letrozole $2.5 \mathrm{mg}$, Sandoz Pharmaceutical Company, Australia) day by day beginning from day 1 of unconstrained (or progesterone initiated) feminine seeping for 10 days (patients, up to 3 cycles).

All patients of the two gatherings observed by trans-vaginal ultrasound for the mean follicular volume and thickness of the endometrium on day 10 of the cycles.

HCG infusion (5,000 IUIM) given when at any rate one follicle estimated $\geq 18 \mathrm{~mm}$.

Patients exhorted for intercourse 24-36h after HCG infusion.

Serum HCG decided fourteen days after HCG infusion without monthly cycle for determination of pregnancy followed by trans vaginal ultrasound for exhibit of the gestational sac.

\section{Results}

This study was conducted on forty patients attending the obstetrics and gynecology Department in Benha University Hospitals as a Prospective randomized comparative study.

General characteristics in both groups

There were no statistically significant differences between both groups regarding the age, weight, and height of patients. $\mathrm{P}$ values were $0.221,0.575$, and 0.637 , respectively.

Table (1) General characteristics in both groups.

\begin{tabular}{llccc}
\hline & & Short protocol(n= 20) & Long protocol(n= 20) & P value \\
\hline Age (years) & Mean \pm SD & $29 \pm 4$ & $31 \pm 3$ & 0.221 \\
& Range & $22-35$ & $25-35$ & 0.575 \\
Weight $(\mathbf{k g})$ & Mean \pm SD & $67 \pm 11$ & $65 \pm 11$ & \\
& Range & $47-88$ & $47-88$ & 0.637 \\
Height $(\mathbf{c m})$ & Mean \pm SD & $157 \pm 9.3$ & $158 \pm 10.6$ & $140-180$ \\
& Range & $140-170$ & & \\
\hline
\end{tabular}

Independent t-test was used 


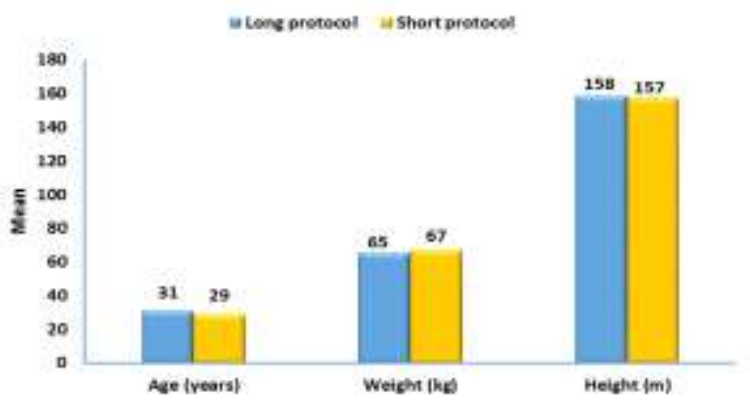

Fig. (1) General characteristics in both groups.

Type of infertility in both groups

Type of infertility showed a non-statistically significant difference between both groups. P-value was 0.519

Table (2) Type of infertility in both groups.

\begin{tabular}{lllccc}
\hline & & & Shortprotocol(n= 20) & Long protocol $(\mathbf{n}=\mathbf{2 0})$ & P value \\
\hline Typeof infertility & 1ry & $\mathbf{n ( \% )}$ & $7(35.0)$ & $9(45)$ & 0.519 \\
& 2ry & $\mathbf{n ( \% )}$ & $13(65)$ & $11(55.0)$ & \\
\hline
\end{tabular}

Chi-square test was used.

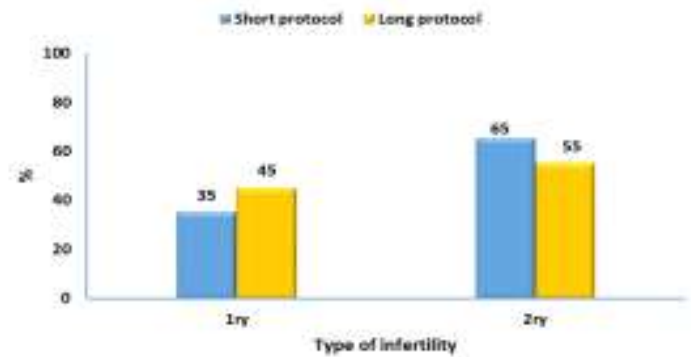

Fig. (2) Type of infertility in both groups

Infertility duration in both groups

Infertility duration showed a non-statistically significant difference between both groups. P-value was 0.675

Table (3) Infertility duration in both groups.

\begin{tabular}{|c|c|c|c|c|}
\hline & & Short protocol $(n=20)$ & Long protocol $(n=20)$ & P value \\
\hline Infertility duration (years) & $\begin{array}{l}\text { Mean } \pm \text { SD } \\
\text { Range }\end{array}$ & $\begin{array}{l}4 \pm 1 \\
2-5\end{array}$ & $\begin{array}{l}3 \pm 1 \\
2-5\end{array}$ & 0.675 \\
\hline
\end{tabular}

Independent t-test was used

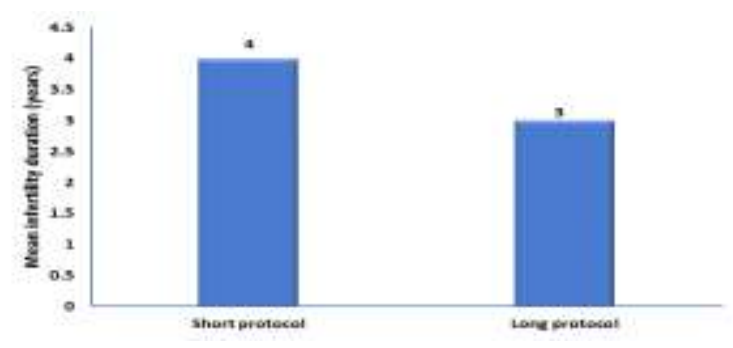

Fig (3) Infertility duration in both groups

Previous induction \& its types in both groups

Previous induction and its types showed non-statistically significant differences between both groups. P values were 1.0 and 0.394 , respectively. 
Table (4) Previous induction \& its types in both groups.

\begin{tabular}{|c|c|c|c|c|c|}
\hline & & & $\begin{array}{l}\text { Short protocol } \\
\quad(n=20)\end{array}$ & $\begin{array}{l}\text { Long protocol } \\
(\mathbf{n}=\mathbf{2 0}) \\
\end{array}$ & $P$ value \\
\hline Previous induction & n (\%) & & $16(80.0)$ & $16(80.0)$ & 1.0 \\
\hline Type of previous induction & $\begin{array}{l}\text { Tablet } \\
\text { Tablet \& iniection }\end{array}$ & $\begin{array}{l}\text { n }(\%) \\
\text { n }(\%)\end{array}$ & $\begin{array}{c}2(12.5) \\
14(87.5)\end{array}$ & $\begin{array}{c}5(31.3) \\
11(68.8)\end{array}$ & 0.394 \\
\hline
\end{tabular}

Fisher's exact test was used

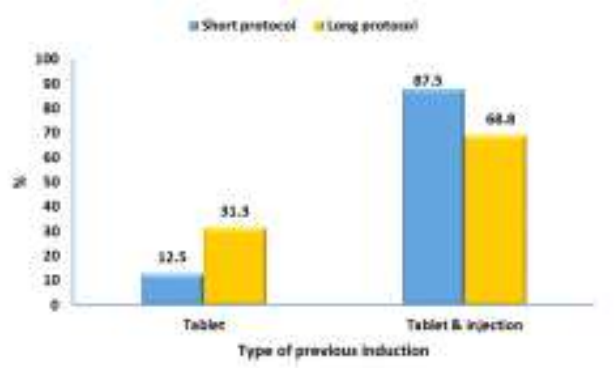

Fig. (4) Type of previous induction in both groups

Number of cycles in both groups

The number of cycles showed a non-statistically significant difference between both groups. P-value was 0.844

Table (5) Number of cycles in both groups.

\begin{tabular}{lllccc}
\hline & & & Short protocol $(\mathbf{n}=\mathbf{2 0})$ & Long protocol(n= 20) & P value \\
\hline Number of cycles & One & $\mathbf{n}(\boldsymbol{\%})$ & $11(55.0)$ & $9(45.0)$ & 0.844 \\
& Two & $\mathbf{n}(\boldsymbol{\%})$ & $5(25.0)$ & $6(30.0)$ & $5(25.0)$ \\
& Three & $\mathbf{n}(\boldsymbol{\%})$ & $4(20.0)$ & & \\
\hline
\end{tabular}

Fisher's exact test was used

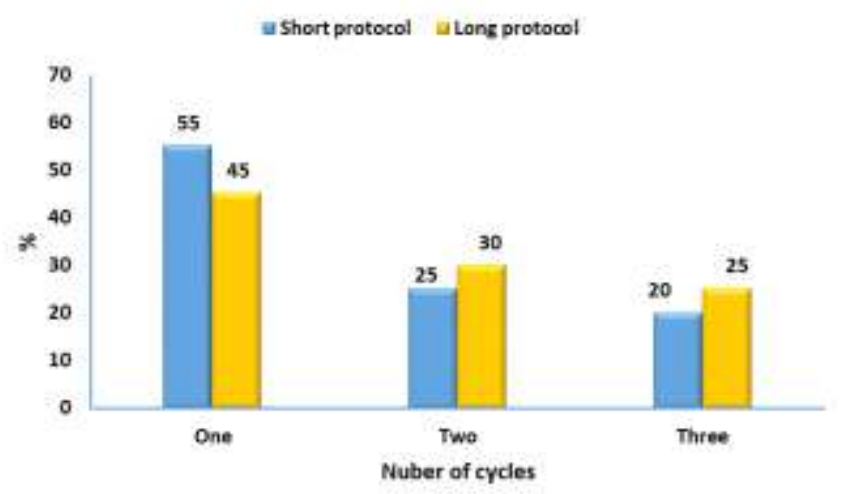

Fig. (5) Number of cycles in both groups

Ultrasound criteria of PCOs in both groups

Ultrasound criteria of PCOs showed a non-statistically significant difference between both groups. P-value was 0.490

Table (6) Ultrasound criteria of PCOs in both groups

\begin{tabular}{lcccc}
\hline & & Short protocol $(\mathbf{n}=\mathbf{2 0})$ & Long protocol $(\mathbf{n}=\mathbf{2 0})$ & P value \\
\hline US criteria of PCOs & $\mathbf{n}(\boldsymbol{\%})$ & $15(75.0)$ & $13(65.0)$ & 0.490 \\
\hline
\end{tabular}

Chi-square test was used 


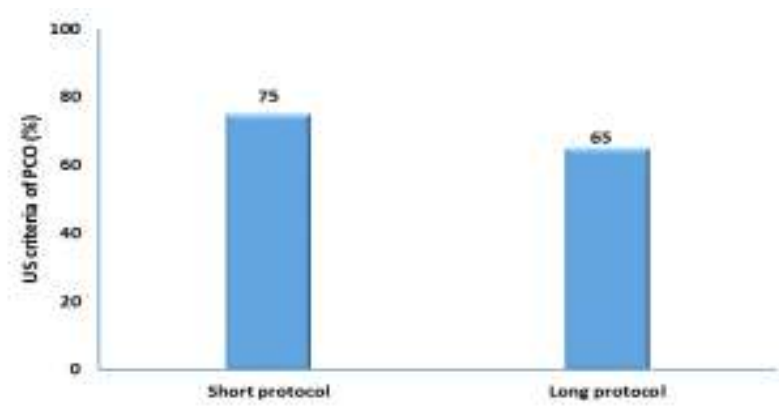

Fig. (6) Ultrasound criteria of PCOs in both groups.

Hyperandrogenism in both groups

Hyperandrogenism showed a non-statistically significant difference between both groups. P-value was 0.525

Table (7) Hyperandrogenism in both groups

\begin{tabular}{lllll}
\hline & & Short protocol $(\mathbf{n}=\mathbf{2 0})$ & Longprotocol $(\mathrm{n}=\mathbf{2 0})$ & P value \\
\hline Hyperandrogenism & $\mathbf{n}(\%)$ & $\mathbf{1 2}(\mathbf{6 0 . 0})$ & $\mathbf{1 0}(\mathbf{5 0 . 0})$ & $\mathbf{0 . 5 2 5}$ \\
\hline
\end{tabular}

Chi-square test was used

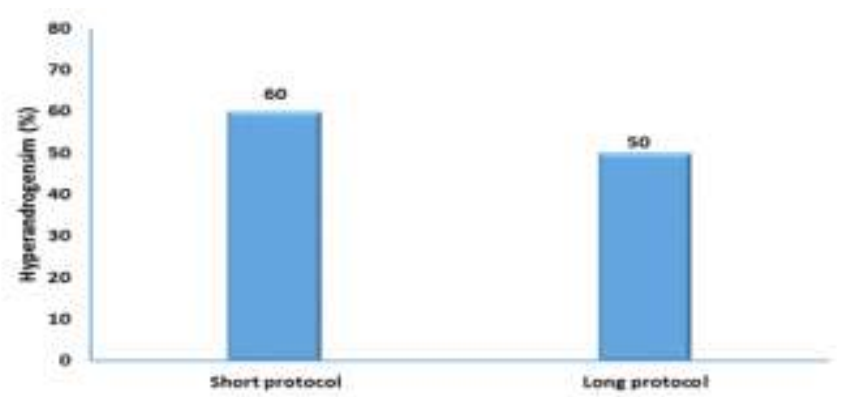

Fig. (7) Hyperandrogenism in both groups

Ovulation in both groups

Ovulation status showed a non-statistically significant difference between both groups. P-value was 1.0

Table (8) Oligo-ovulation in both groups

\begin{tabular}{llccc}
\hline & & Short protocol $(\mathbf{n}=\mathbf{2 0})$ & Long protocol $(\mathbf{n}=\mathbf{2 0})$ & P value \\
\hline Ovulation & Anovulation & $9(45.0)$ & $7(35.0)$ & 0.519 \\
& Oligo-ovulation & $11(55.0)$ & $13(65.0)$ & \\
\hline
\end{tabular}

Chi-square test was used

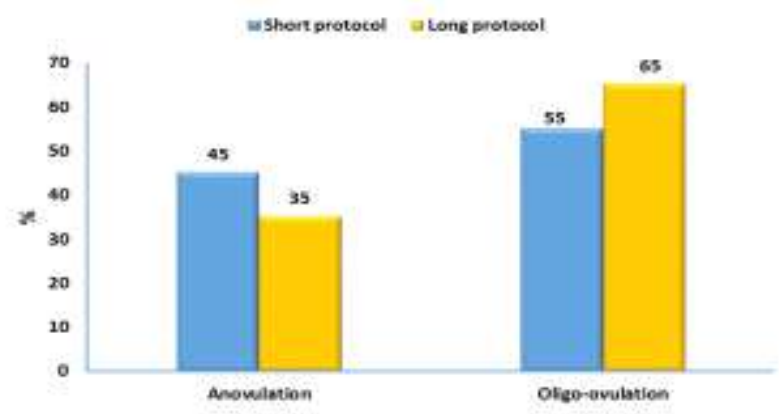

Fig. (8) Ovulation in both groups.

Levels of FSH, LH, and testosterone in both groups

Levels of FSH, LH, and testosterone showed non-statistically significant differences between both groups. $\mathrm{P}$ values were $0.257,0.667$, and 0.946 , respectively. 
Table (9) Levels of FSH, LH, and testosterone in both groups.

\begin{tabular}{llccc}
\hline & & Short protocol $(\mathbf{n}=\mathbf{2 0})$ & Long protocol $(\mathbf{n}=\mathbf{2 0})$ & P value \\
\hline \multirow{2}{*}{ FSH } & Mean \pm SD & $5.7 \pm 1.7$ & $6.4 \pm 2.1$ & 0.257 \\
& Range & $3.5-9$ & $3.4-9.5$ & 0.667 \\
LH & Mean \pm SD & $10.6 \pm 4.4$ & $11.1 \pm 4$ & 6.19 \\
\multirow{2}{*}{ Testosterone (nmol/l) } & Range & $4.5-19$ & $2.7 \pm 0.7$ & 0.946 \\
\hline
\end{tabular}

Independent t-test was used

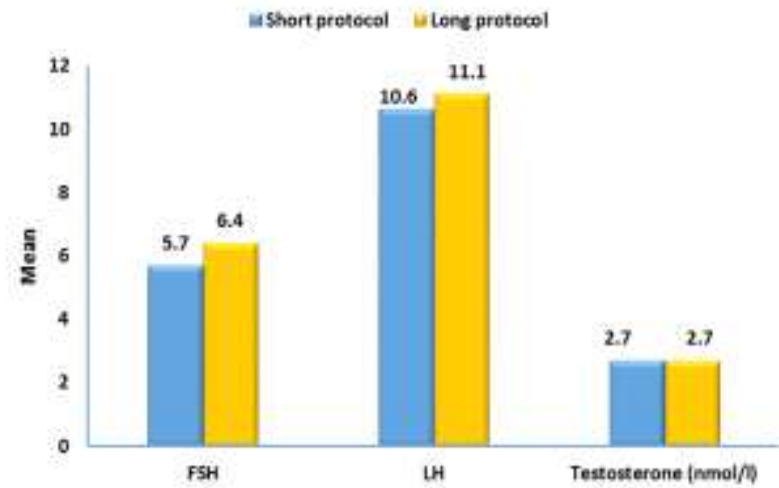

Fig. (9) Levels of FSH, LH, and testosterone in both groups.

Total number of follicles in both groups

The mean total number of follicles was precycle of ovulation induction significantly higher in the long protocol group (4) than the short protocol group (3). P-value was $<0.001$.

Table (10) Total number of follicles in both groups.

\begin{tabular}{lllll}
\hline & & Short protocol $(\mathbf{n}=\mathbf{2 0})$ & Long protocol $(\mathbf{n}=\mathbf{2 0})$ & P value \\
\hline Total N of follicles & Mean $\mathbf{E S D}$ & $3 \pm 1$ & $4 \pm 1$ & $<0.001$ \\
& Range & $2-3$ & $3-5$ & \\
\hline
\end{tabular}

Independent t-test was used

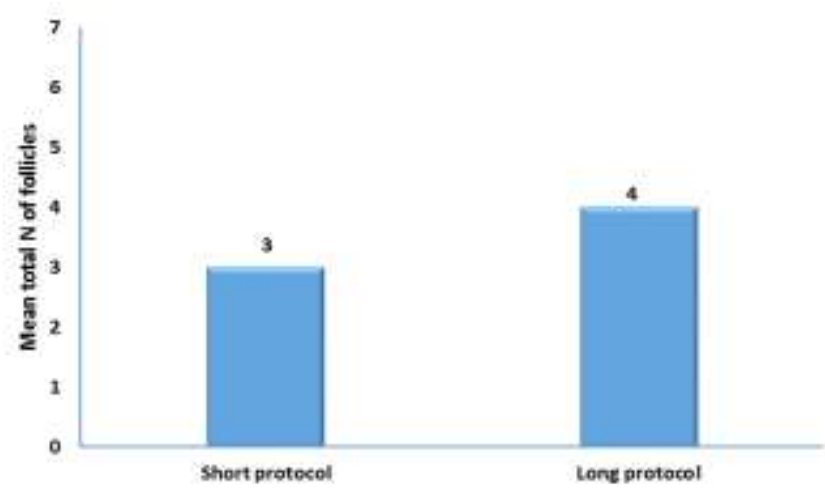

Fig. (10) Total number of follicles in both groups.

Dominant follicle size in both groups

Mean dominant follicle size was larger in the long protocol group (18 $\mathrm{mm})$ than the short protocol group (17 $\mathrm{mm})$, with no statistical significance. P-value was 0.476 . 
Table (11) Dominant follicle size in both groups.

\begin{tabular}{llccc}
\hline & & Short protocol $(\mathbf{n}=\mathbf{2 0})$ & Long protocol $(\mathbf{n}=\mathbf{2 0})$ & P value \\
\hline Dominant follicle size $(\mathbf{m m})$ & Mean $\mathbf{\pm S D}$ & $17 \pm 4$ & $18 \pm 4$ & 0.476 \\
& Range & $12-25$ & $12-25$ & \\
\hline
\end{tabular}

Independent t-test was used

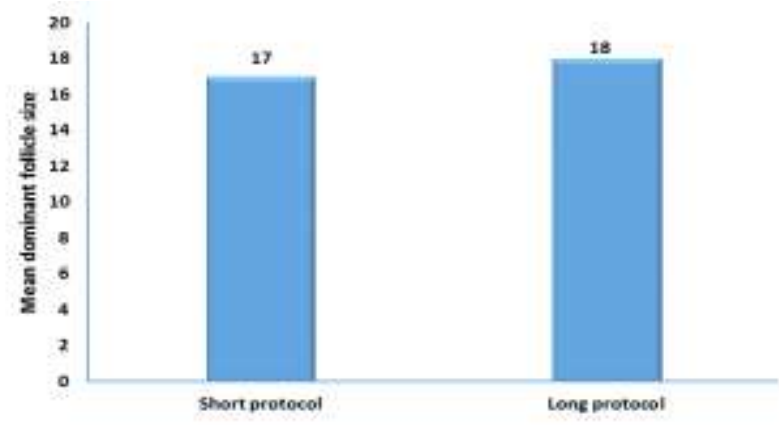

Fig. (11) Dominant follicle size in both groups

Dominant follicle size categories in both groups

Follicle size $<14 \mathrm{~mm}$ was higher in short protocol (40.0\%) than long protocol (30.0\%), follicle size of 14 to $17 \mathrm{~mm}$ was higher in long protocol (15.0\%) than short protocol (10.0\%), and follicle size of $\geq 18 \mathrm{~mm}$ was higher in long protocol $(55.0 \%)$ than short protocol $(50.0 \%)$, with no statistical significance $(\mathrm{P}$ value $=0.766)$

Table (12) Dominant follicle size categories in both groups.

\begin{tabular}{lllccc}
\hline & & & Short protocol $(\mathbf{n}=\mathbf{2 0})$ & Long protocol $(\mathbf{n}=\mathbf{2 0})$ & P value \\
\hline Dominant follicle size & $<\mathbf{1 4}$ & $\mathbf{n}(\boldsymbol{\%})$ & $8(40.0)$ & $6(30.0)$ & 0.766 \\
& $\mathbf{1 4}$ to $\mathbf{1 7}$ & $\mathbf{n}(\boldsymbol{\%})$ & $2(10.0)$ & $3(15.0)$ & $11(55.0)$ \\
& $\geq \mathbf{1 8}$ & $\mathbf{n}(\boldsymbol{\%})$ & $10(50.0)$ & & \\
\hline
\end{tabular}

Chi-square test was used

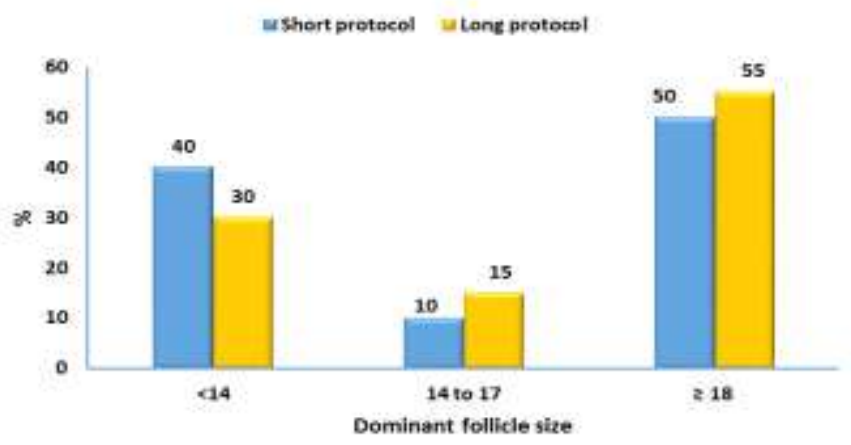

Fig. (12) Dominant follicle size categories in both groups.

Endometrial thickness at the time of HCG administration size in both groups

Endometrial thickness at the time of HCG administration showed a non-statistically significant difference between both groups. P-value was 0.713

Table (13) Endometrial thickness at the time of HCG administration size in both groups.

\begin{tabular}{|c|c|c|c|c|}
\hline & & $\begin{array}{l}\text { Short protocol } \\
(\mathbf{n}=20)\end{array}$ & $\begin{array}{l}\text { Long protocol } \\
\quad(\mathbf{n}=\mathbf{2 0})\end{array}$ & P value \\
\hline Endometrial thickness at time of HCG (mm) & $\begin{array}{l}\text { Mean } \pm \text { SD } \\
\text { Range }\end{array}$ & $\begin{array}{c}8 \pm 2 \\
5-11\end{array}$ & $\begin{array}{c}8 \pm 2 \\
5-11\end{array}$ & 0.713 \\
\hline
\end{tabular}

Independent t-test was used 


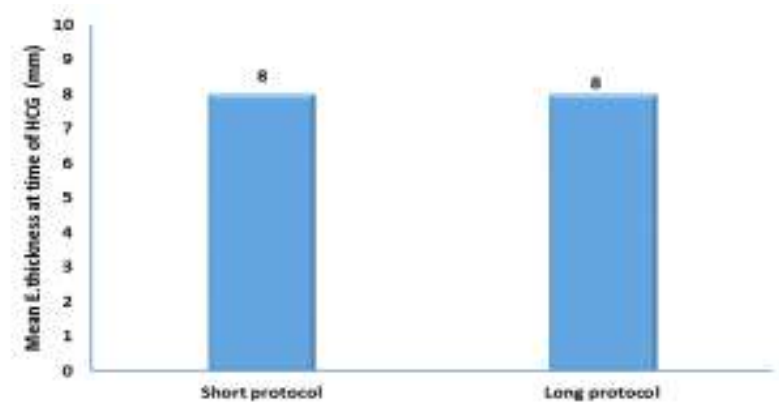

Fig. (13) Endometrial thickness at time of HCG administration size in both groups.

The occurrence of ovulation in both groups

Ovulation occurrence was higher in the long protocol group $(65.0 \%)$ than the short protocol group $(60.0 \%)$, with no statistical significance. P-value was 0.744

Table (14) Occurrence of ovulation in both groups.

\begin{tabular}{lllll}
\hline & & Short $\operatorname{protocol}(\mathbf{n}=\mathbf{2 0})$ & Long protocol $(\mathbf{n}=\mathbf{2 0})$ & P value \\
\hline Ovulation occurrence & $\mathbf{n}(\boldsymbol{\%})$ & $12(60.0)$ & $13(65.0)$ & 0.744 \\
\hline
\end{tabular}

Chi-square test was used

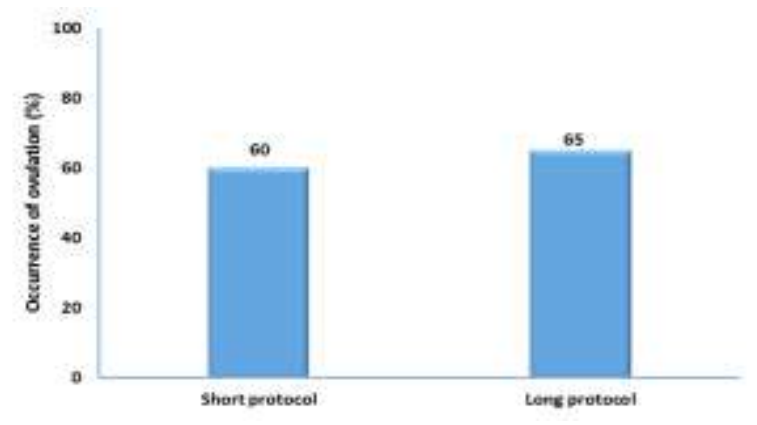

Fig. (14) Occurrence of ovulation in both groups.

The occurrence of pregnancy \& miscarriage in both groups

The occurrence of pregnancy was higher in the long protocol group (25.0\%) than the short protocol group (15.0\%), with no statistical significance. P-value was 0.695

Table (15) Occurrence of pregnancy \& miscarriage in both groups

\begin{tabular}{lcccc}
\hline & & Short protocol $(\mathbf{n}=\mathbf{2 0})$ & Long protocol $(\mathbf{n}=\mathbf{2 0})$ & P value \\
\hline Occurrence of pregnancy & $\mathbf{n}(\boldsymbol{\%})$ & $3(15.0)$ & $5(25.0)$ & 0.695 \\
Miscarriage* & $\mathbf{n}(\%)$ & $0(0.0)$ & $1(20.0)$ & - \\
\hline
\end{tabular}

Fisher's exact test was used

* Percentage was calculated based on total pregnancies

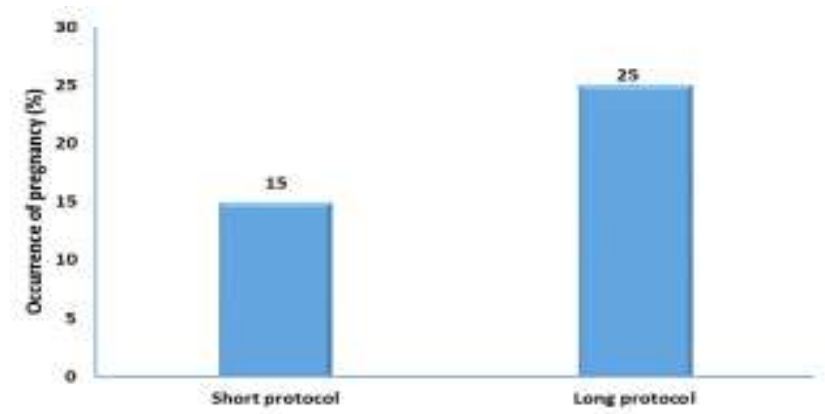

Fig. (15) Occurrence of pregnancy in both groups. 


\section{Discussion}

PCOS is a typical endocrine issue, It is related with constant anovulation, and causes barrenness in $4 \%-6 \%$ of ladies in the regenerative age group.Despite the wide acknowledgment of clomiphene citrate as afirst-line drug for ovulation enlistment in ladies with PCOS, a signifcant extent of ladies don't react to this treatment [3].

Different administrations were proposed for barren ladies with PCOS $(15,17,18)$. In any case, the ideal administration alternative has not been tended to fulfilled. Albeit different medicines including weight decrease, clomiphene citrate, metformin, gonadotropins, and ovary searing have been accounted for to treat such condition, the viability actually has deficient proof to help $[8,11]$.

Past investigation had proposed that letrozole, a third-age aromatase inhibitor, can be utilized for ovulation acceptance, and it is related with a higher possibility of simple pregnancy and a lower hazard of various pregnancy than clomiphene citrate.

The current investigation was done at Benha University Hospital out-patient center of obstetric \&gynecology to analyze between short $(5 \mathrm{mg}$ day by day for 5 days) versus broadened course $(2.5 \mathrm{mg}$ day by day for 10 days) of letrozole treatment for ovulation enlistment in ladies with polycystic ovary condition.

This planned similar examination included 40 fruitless ladies who were analyzed as having PCOS. The remembered ladies for this examination were separated into two gatherings. Gathering I was initiated to ovulate by short course. Gathering II was initiated to ovulate by broadened course.

In the current investigation, the outcomes were as following:

- Age dissemination among bunch I (short letrozole bunch) was $22 \mathrm{y}$ to $35 \mathrm{y}(29 \pm 4)$ and among bunch II (long letrozole bunch) was $25 y$ to $35 y(31 \pm 3)$.

- Number of Oligo-ovulating patients was more noteworthy in the long letrozole bunch contrasted with short letrozole bunch (65\% versus $55 \%)$. separately.

- Number of patients with Hyper-androgenism among groupI (short letrozole) was $60 \%$ and bunch II (long letrozole)was $60 \%$.

- Percentage of patients with positive US information was $75 \%$ in short letrozole bunch and $75 \%$ in long letrozole bunch.

- The mean worth \pm SD for complete number of follicles after incitement was altogether more prominent in the long letrozole bunch $(4 \pm 1)$ versus $(3 \pm 1)$ in short letrozole group; $\mathrm{P}=<0.001)$

- This outcome come in concur with the consequence of study done by Yadav,(16))that was done to assess the viability - on richness result by present moment letrozole versus broadened letrozole routine in clomiphene citrate safe PCOS ladies, directed on 105 ladies with clomiphene safe PCOS, Group 1 (55 patients) got short letrozole routine, bunch 2 ( 50 patients) got long letrozole routine

- Their outcomes - in this point - were that the absolute number of follicles during incitement was altogether more noteworthy in the long letrozole bunch $(6.8+1$ .3 versus $3.5+/-.5$ )

- The consequences of our examination were in concur with the aftereffects of the investigation by Al Fadhli (2)which showed that the ovulation rate and pregnancy rate was 1.9 and $18 \%$ separately in the gathering who got letrozole in a portion of $20 \mathrm{mg}$ while it was lower i.e. 1.7 and $15 \%$ in the gathering who got letrozole in a portion of $12.5 \mathrm{mg}$. On contrasting the two gatherings in the short letrozole bunch the pregnancy rate was $12.5 \%$ and the unnatural birth cycle rate was $16.7 \%$ while in the allencompassing letrozole bunch it was $18.3 \%$ and $11.1 \%$ separately These outcomes were reliable to the investigation directed by Badawy and Alaa Mosbah [4] in which the pregnancy rate was $12.4 \%$ and the premature delivery rate was $18.4 \%$ in the short letrozole bunch while it was $17.4 \%$ and $17.9 \%$ in the long letrozole bunch individually

- These outcomes likewise come concur with the consequences of study done by Badawy (4) that was done to assess the result of long letrozole treatment for enlistment of ovulation in patients with clomiphene-safe polycystic ovary disorder (PCOS) on 218 patients with clomiphene-safe PCOS and patients were arbitrarily assigned to treatment with either long letrozole treatment $(n=108)$ or short letrozole treatment $(n=110)$. Their outcomes in this point were the absolute quantities of follicles during incitement was essentially more prominent in the long letrozole bunch $(6.7+/-0.3$ versus $3.9+/-0.4)$.

- Another examination was conveyed by Fouda (6)on 136 ladies who reacted ineffectively to GnRH agonist long convention in their first IVF cycle and were randomized into two equivalent gatherings utilizing PC produced list and were treated in the second IVF cycle by either expanded letrozole routine $(5 \mathrm{mg} /$ day during the initial 5 days of cycle and $2.5 \mathrm{mg} /$ day during the resulting 3 days) joined with HPuFSHGnRHant convention or short letrozole routine (2.5 $\mathrm{mg}$ /day from cycle day 3-7) joined with HPuFSHGnRHant convention. The examination was done to think about the viability and cost-adequacy of broadened high portion letrozole routine/HPuFSHgonadotropin delivering chemical opponent (GnRHant) convention with short low portion letrozole routine/HPuFSH-GnRHant convention in helpless responders going through IVF-ET.The study showed that there were no huge contrasts between the two gatherings concerning number of oocytes recovered in expanded letrozole bunch and in short letrozole bunch $(5.39 \pm 2.08$ versus $5.20 \pm 1.88)$, separately.

- Another examination was finished by Ramezanzadeh (5)to think about the impacts of either a 5 or $7.5 \mathrm{mg}$ 
every day portion of Letrozole in PCOS ladies going through ovulation acceptance. 67 PCOS patients with barrenness were haphazardly isolated into two gatherings and treated with either $5 \mathrm{mg} /$ day $(30$ patients, bunch 1) or $7.5 \mathrm{mg} /$ day (37 patients, bunch 2) Letrozole, for 5 days beginning from day 3 of the monthly cycle. The outcome showed no huge contrast in the quantity of intermediate $(0.83 \pm 0.75$ versus $0.62 \pm 0.76)$ and develop follicles $(1.13 \pm 1.11$ versus $1.22 \pm 1.03$ ) on days $12-14$ between bunch 1 and 2 , individually.

- In the current examination, the mean size $(\mathrm{mm})$ of greatest follicles was more prominent $(18 \pm 4)$ in the long letrozole bunch when contrasted with short letrozole bunch $(17 \pm 4)$, without measurable contrasts $(\mathrm{P}=0.476)$.

- In the current investigation, the quantity of patients with follicles estimating $\geq 18 \mathrm{~mm}$ and between 14$17 \mathrm{~mm}$ was more prominent in the long letrozole bunch $(\mathrm{P}=0.766)$. These outcomes came in similar with the consequences of Badawy (4)

- In the current examination, The mean of endometrial thickness at the hour of hCG organization \pm SD in bunch I was $8 \pm 2 \mathrm{~mm}$ and $8 \pm 2$ among bunch II. There was no critical contrast in the endometrial thickness at the hour of hCG organization between the two gatherings $(\mathrm{P}=0.713)$.

- That came concur with Badawy (4)and Ramezanzadeh(5).

- In the current examination, level of ovulation occured after treatment, bunch II (long letrozole bunch) was more noteworthy than Group I (short letrozole bunch) (65\% versus $60 \%$, individually).

In the current examination, the mean size $(\mathrm{mm})$ of greatest follicles was more prominent $(18 \pm 4)$ in the long letrozole bunch when contrasted with short letrozole bunch $(17 \pm 4)$, without measurable contrasts $(\mathrm{P}=0.476)$.

In the current investigation, the quantity of patients with follicles estimating $\geq 18 \mathrm{~mm}$ and between $14-17 \mathrm{~mm}$ was more prominent in the long letrozole bunch $(\mathrm{P}=0.766)$. These outcomes came in similar with the consequences of Badawy [4].

In the current examination, The mean of endometrial thickness at the hour of hCG organization \pm SD in bunch $\mathrm{I}$ was $8 \pm 2 \mathrm{~mm}$ and $8 \pm 2$ among bunch II. There was no critical contrast in the endometrial thickness at the hour of hCG organization between the two gatherings $(\mathrm{P}=0.713)$.

That came concur with Badawy [4] and Ramezanzadeh [5].

In the current examination, level of ovulation occured after treatment, bunch II (long letrozole bunch) was more noteworthy than Group I (short letrozole bunch) (65\% versus $60 \%$, individually).

\section{Conclusion}

The long letrozole treatment ( $2.5 \mathrm{mg}$ for 10 days) can deliver more develop follicles and thusly a greater number of pregnancies than the short letrozole treatment ( $5 \mathrm{mg}$ for 5 days).

\section{Reference}

[1] R.Azziz, K.Kintziger, R.Li, J.Laven, L.MorinPapunen, SS.Merkin, H.Teede, BO.Yildiz. Recommendations for epidemiologic and phenotypic research in polycystic ovary syndrome: an androgen excess and PCOS society resource. Hum Reprod. Nov 1.vol.34(11),pp.2254-2265,2019.

[2] R.Al Fadhli ,C.Sylvestre, W.Buckett . A randomized trial of superovulation with two different doses of letrozole .Fertil Steril.vol.85,pp.161 $164,2006$.

[3] V.Atay, C.Cam, M.Cam, A.Karateke. Comparison of letrozole and clomiphene citrate in women with polycystic ovaries undergoing ovarian stimulation. J Int Med Res.vol. 34,pp.73-76,2006.

[4] A.Badawy, Aal IA, M. Abulatta . Clomiphene citrate or Letrozole for ovulation induction in women with polycysiic ovarian syndrome: a prospective rundomized trial. Fertil Steril. Vol.92(3),pp.849$852,2009$.

[5] Fatemeh Ramezanzadeh, Roya Nasiri, Mohammad Sarafraz Yazdi, Maryam Baghrei. A randomized trial of ovulation induction with two different doses of Letrozole in women with PCOS.vol.284,pp.1029-1034,2011.

[6] UM.Fouda, AM.Sayed. Extended letrozole regimen versus clomiphene citrate for superovulation in patients with unexplained infertility undergoing intrauterine insemination: A randomized controlled trial. Reprod Biol Endocrinol.vol.9,pp.84,2011.

[7] L.Gibson, D.Lawrence, C.Dawson, J.Bliss. Aromatase inhibitors for treatment of advanced breast cancer in postmenopausal women. Cochrane Database Syst Rev. Oct .vol.7,pp. (4):CD003370,2009.

[8] PR.Jirege, RS.Patill. Comparison of endocrine and ultrasound profiles during ovulation induction with clomiphene citrate and letrozole in ovulatory volunteer women. Fertil Steril.vol.93,pp.17483,2010 .

[9] MP.Lauritsen, JG.Bentzen, A.Pinborg,. The prevalence of polycystic ovary syndrome in a normal population according to the Rotterdam criteria versus revised criteria including anti-mullerian hormone. Hum Reprod.vol.29(4),pp.791,2014.

[10] S.Mitra, PK.Nayak, S.Agarwal. Laproscopic ovarian drilling: An alternative but not an ultimate in the management of polycystic ovary syndrome. J Nat Sc Bial Med .vol. 6,pp. 40-8. ,2015.

[11] AA.Rouzi, MS.Ardawi. Arandomized controlled trial of the efficacy of rosiglitazone and clomiphene citrate versus metformin and clomiphene citrate in women with clomiphene citrate-resistant polycystic ovary syndrome. Fertil Steril.vol.85,pp.428-35,2006.

[12] K.Sujata, S.Swoyam. 2D and 3D Transvaginal Sonography to Determine Cut-offs for 
Ovarian Volume and Follicle Number per Ovary for Diagnosis of Polycystic Ovary Syndrome in Indian Women. J Reprod Infertil.vol.19(3),pp.146-151,2018.

[13] Simone Brixius-Anderko, E.Emily. Scott, Structure of human cortisol-producing cytochrome P450 11B1 bound to the breast cancer drug fadrozole provides insights for drug design, Journal of Biological Chemistry .vol. 294, pp. 453-460, ISSN 0021-9258,2019.

[14] P.Thomas. Ahern, Daniel L.Hertz, Per Damkier, Bent Ejlertsen, Stephen J.Hamilton-Dutoit, James M.Rae, Meredith M.Regan, Alastair M.Thompson, Timothy L.Lash, Deirdre P.CroninFenton, Cytochrome P-450 2D6 (CYP2D6) Genotype and Breast Cancer Recurrence in Tamoxifen-Treated Patients: Evaluating the Importance of Loss of Heterozygosity, American Journal of Epidemiology.vol. 185, pp. 75-85, 2017.

[15] J.Wang, L.Zhu, K.Hu,. Effects of metformin treatment on serum levels of C-reactive protein and interleukin-6 in women with polycystic ovary syndrome: a meta-analysis: a PRISMA-compliant article. Medicine (Baltimore).vol.96,pp.e8183,2017.

[16] P.Yadav, M.Agarwal, V.Agarwal, U.Verma, R.Agarwal. To Study the Efficacy on Fertility Outcome by short term Letrozole versus Extended Letrozole Regimen in Clomiphene Citrate Resistant PCOS Women.vol.8,pp.22-45,2018.

[17] Zeng, Xian-Ling $\mathrm{MD}^{\mathrm{a}}$; Zhang, Ya-Fei $\mathrm{MD}^{\mathrm{b}}$; Tian, Quan $\mathrm{PhD}^{\mathrm{a}}$; Xue, Yan $\mathrm{PhD}^{\mathrm{a}}$; An, Rui-Fang

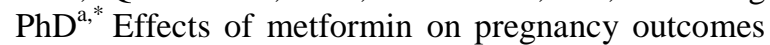
in women with polycystic ovary syndrome, Medicine: September.vol. 95 -Issue 36,pp e4526 ,2016.

[18] J.Zhou, L.Yang, J.Yu, Efficacy of acupuncture on menstrual frequency in women with polycystic ovary syndrome: Protocol for a randomized, controlled trial. Randomized controlled trial. Medicine (Baltimore).vol.96,pp.e8828,2017. 